\title{
The Enactivist Revolution
}

\author{
Kenneth Aizawa \\ Department of Philosophy \\ Rutgers University, Newark \\ ken.aizawa[]gmail.com \\ Received December 2013; accepted June 2014; published Autumn 2014.
}

\begin{abstract}
Among the many ideas that go by the name of "enactivism" there is the idea that by "cognition" we should understand what is more commonly taken to be behavior. For clarity, label such forms of enactivism "enactivismb." This ter-

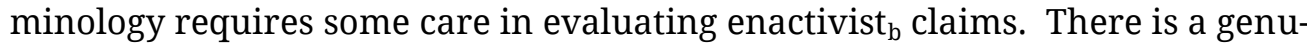
ine risk of enactivist and non-enactivist cognitive scientists talking past one another. So, for example, when enactivists b $_{b}$ write that "cognition does not require representations" they are not necessarily denying what cognitivists claim when they write that "cognition requires representations." This paper will draw attention to instances of some of these unnecessary confusions.
\end{abstract}

Keywords: enactivism; enaction; cognition; behavior; autopoiesis.

In soliciting contributions to this special issue of Avant, the editors asked whether enactivism fits cognition. This question, however, may well misinterpret what at least some forms of enactivism are about. It may underestimate the breadth of the revolution that at least some strains of enactivism are championing. For some in the enactivist movement, it appears that the goal is not merely to provide a revolutionary new account of what cognition is. We need no more of that. What is needed, instead, is a cognitive science that studies something else. What is needed is a cognitive science that does not study cognition! That would be a real revolution.

But, what, one might ask, would such a really revolutionary cognitive science study, if not cognition? One popular proposal is that it should study what has been, and generally continues to be, known as (a type of) behavior. ${ }^{3}$ For con-

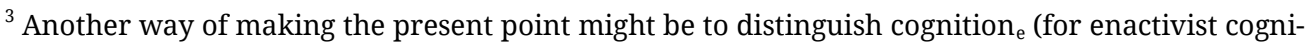
tion) and $\operatorname{cognition}_{\mathrm{c}}$ (for cognitivist cognition). This might make it easier to see that this issue is
} 
venience, and in order to distinguish this form of enactivism from other forms, let us label it "enactivism (a type of) behavior. To be sure, not all those who think of themselves as enactivists are enactivists $\mathrm{b}_{\mathrm{b}}$. Nevertheless, there are prominent representatives of enactivism ${ }_{b}$. To take one salient example, many enactivists $\mathrm{b}_{\mathrm{b}}$ have been inspired, at least to some degree, by Humberto Maturana and Francisco Varela's Autopoiesis and Cognition. Maturana is relatively explicit about understanding a cognitive system as a (self-maintaining) behaving system. According to Maturana, "A cognitive system is a system whose organization defines a domain of interactions in which it can act with relevance to the maintenance of itself, and the process of cognition is the actual (inductive) acting or behaving in this domain." (Maturana and Varela 1980: 13). To take a more recent example, Tony Chemero proposes, "cognitive scientists ought to try to understand cognition as intelligent behavior" (Chemero 2009: 25). Further, he believes that "radical embodied cognitive science can explain cognition as the unfolding of a brain-body-environment system" (Chemero 2009: 43). But, "the unfolding of a brain-body-environment system" sounds like a metaphor for behavior.

The foregoing point might be made in another way. Notice that enactivists often propose their theory as an alternative to cognitivism, but they are not always explicit about what in the cognitivist view they reject. As one example, Stewart, Gapenne, and Di Paolo write,

The aim of this book is to present the paradigm of enaction as a framework for a far-reaching renewal of cognitive science as a whole. There have been many critiques of classical, first-generation cognitivism based on the Computational Theory of Mind. A distinctive feature of this book is a deliberate choice not to go over that old ground yet again, but to reserve the energy for positive exploration of new paths. (Stewart, Gapenne, and Di Paolo 2010: vii).

Di Paolo, Rohde, and De Jaegher 2010, provide a similarly opened-ended rejection of cognitivism:

\begin{abstract}
Almost two decades since the publication of The Embodied Mind (Varela, Thompson, and Rosch 1991), the term enactive has moved out of relative obscurity to become a fashionable banner in many regions of cognitive science. ... Theirs was not only an achieved synthesis of existing criticisms to a predominantly computationalist paradigm, but also the articulation of a set of postulates to move these ideas forward. Indeed, the increasing use of enactive terminology serves as an indication that the time is ripe for a new era in cognitive science. To a great extent, we believe this to be so. (Di Paolo, Rohde, and De Jaegher 2010: 33)
\end{abstract}

not about the "right" way to use "cognition" or who gets to use it how. Those who prefer to use this terminology are free to use it, but this paper will adhere to the more mainstream usage. The important point, of course, is not ultimately about the terminology, but the fact that cognitivists and some enactivists are talking about two different things. 
One might well think that enactivism ${ }_{b}$ means to displace the cognitivist's computation theoretic apparatus of rules and (especially) representations with another sort of apparatus. This alternative apparatus might be the mathematics of dynamical systems theory or one or another definition of autopoiesis. In such a vision, enactivism ${ }_{b}$ and cognitivism are competing theories of the same thing in the way that Newton's theory of gravitation and Einstein's theory of general relativity were competing theories of a single putative force in nature, namely, gravity. This, however, apparently underestimates just how sweeping a change the enactivists ${ }_{b}$ wish to make in cognitive science. Enactivists $_{b}$ generally propose to walk away from the issues and concerns of mainstream cognitive science to focus on what is commonly understood as behavior. In practice, therefore, enactivists ${ }_{b}$ use different tools to study different issues.

While there are times when enactivists $s_{b}$ enthusiastically embrace the dramatic changes implicit in their work, they also tend to paper over the significance of the proposal to study (a type of) behavior by calling behavior "cognition" or "lower-level cognition" or "basic cognition" or "minimal cognition." (See, for example, Calvo and Keijzer 2009, Chemero 2009, Stewart 2010, Di Paolo, Rohde, and De Jaegher 2010, and Hutto and Myin 2013.) This terminology is likely to be misleading to mainstream cognitive scientists, but a more serious problem is that it seems to mislead even some enactivists $\mathrm{b}_{\mathrm{b}}$. There are times when they write as if they intend to address traditional, sometimes longstanding, problems surrounding cognition. Yet, because they use "cognition" as a term for (a type of) behavior, they are thereby not talking about the same thing as are the traditional cognitivists. Thus, they sometimes fail to come to grips with traditional issues in cognitive science.

This paper will begin, in section 1, with a brief review of the distinction between cognition and behavior as it has formerly been used in cognitive science, namely, that cognitive processes have been thought to be among the many endogenous factors that contribute to the production of behavior. The point here is not to offer definitions of "cognition" or "behavior" or to offer much in the way of clarification of what each of these is, but simply to draw attention to what has been a widely held understanding of the difference between the two. Successive sections (sections 2-5) will then review ways in which Stewart 2010, Froese, Gershenson, and Rosenblueth 2013, and Hutto and Myin 2013, seem not to appreciate the significance of their departure from traditional problems of cognition. Section 6 will emphasize the fact that not all enactivists are enactivists ${ }_{b}$ by providing clear examples of enactivists who offer a more traditional conception of cognition as a species of endogenous cause of behavior. 
As a final preliminary note, it is important to bear in mind that the goal of this paper is clarification, not criticism. Enactivism ${ }_{b}$ clearly represents a dramatic break with tradition. Enactivists have made that abundantly clear. What bears greater attention, however, is the character of this break. Rather than examine endogenous causes of behavior, such as cognition, enactivism ${ }_{b}$ proposes to focus on (a type of) behavior. What also bears attention are some of the ramifications of this break. Insofar as enactivism $b$ no longer addresses cognition as it has formerly been understood, it just so far threatens to ignore cognition. Enactivism en $_{\mathrm{b}}$ thus, does not so much solve traditional problems, as merely walks away from them. This, of course, does not bear directly on the truth of enactivism ${ }_{b}$. It only suggests that enactivists $\mathrm{b}_{\mathrm{b}}$ need to be more careful in how they deal with traditional problems. If they want to talk about traditional cognition, they apparently need an account of endogenous influences on behavior. Alternatively, if they wish to break with tradition, then they must be careful to make a cleaner break. So, to repeat, the goal of this paper is not so much criticism of enactivism ${ }_{b}$ as clarification.

\section{Cognition and Behavior}

One way in which to appreciate the core theoretical commitments of traditional cognitive science might be to revisit some of its founding documents, wherein the original commitments are articulated. As an illustration of this method, Aizawa 2014, describes a bit of common ground between B. F. Skinner and Noam Chomsky, namely, both believed that cognition was a putatively explanatory causal factor in the production of behavior. Where Skinner and Chomsky differed, of course, was in their assessment of the genuine explanatory value of the cognitive. (See, for example, Skinner 1957, and Chomsky 1959.) Another paper that illustrates the core theoretical commitments of traditional cognitive science is the seminal 1958 paper by Alan Newell, J. C. Shaw, and Herbert Simon, "Elements of a Theory of Human Problem Solving.” They propose that

Questions about problem-solving behavior can be answered at various levels and in varying degrees of detail. The theory to be described here explains problem-solving behavior in terms of what we shall call information processes. If one considers the organism to consist of effectors, receptors, and a control system for joining these, then this theory is mostly a theory of the control system. It avoids most questions of sensory and motor activities. (Newell, Shaw, and Simon 1958: 151).

This brief passage contains a number of ideas that are relevant to understanding the differences between the traditional information processing approach in cognitive science and enactivism $b$. These features are worth reviewing in detail. 
The very first sentence proposes to treat problem solving as a behavior. Problem solving is, thus, not itself information processing or cognitive processing. In the literature on embodied and enactive cognition, one sometimes encounters the idea that problem solving is not behavior, but is instead cognitive processing. This claim is more complicated than one might expect. It contains a subtle ambiguity. There is one sense of this claim that is entirely uncontroversial and consistent with the Newell, Shaw, and Simon perspective. It seems perfectly reasonable to claim that the entire process of, say, physically manipulating pencil and paper to solve a cryptarithmetic problem counts as problem solving and cognitive processing. This is the sense in which the whole of the process is cognitive processing in virtue of the fact that an important or salient component of the process is cognitive processing. The whole of the manipulative process is cognitive processing, even though strictly speaking only a proper part of the process is cognitive processing. The idea here might be understood through an analogy. The whole of the process of baking a cake, one might say, is not strictly speaking a matter of baking a cake. The process of baking a cake might include breaking some eggs and the mixing of ingredients, processes that are not themselves baking processes strictly speaking. Similarly, the whole of the process of filling up one's car is not strictly speaking a matter of pumping gasoline into the tank. It includes such things as slowing the car, pulling it into the station, and shutting off the engine. In contrast to this unproblematic claim there is the idea that, strictly speaking, the whole of the process of manipulating the pencil and paper is cognitive processing. This would be the sense in which the whole of the process of baking a cake is literally the baking of a cake or the whole of the process of filling one's gas tank is pumping gasoline into the tank. What probably obscures the ambiguity in the claim that problem solving is cognitive processing is the relative lack of clarity about the character of the component processes. There is a relatively clear distinction between slowing the car to pull it into the gas station and pumping the gasoline into the tank, but it is less clear how to distinguish the information processing that might take place only in the brain and what might be called the information processing that takes place in the pencil and paper. It is, therefore, useful in discussing such cases to be clear on the strength of the claim that problem solving is cognitive processing. The claim is subtler than one might have expected.

Second, the passage from Newell, Shaw, and Simon treats behavior as distinct from cognitive or information processing. Information processing is taken to be a mechanism realized in the brain. This flatly contradicts the enactivist ${ }_{b}$ idea that cognition is (a type of) behavior.

Third, Newell, Shaw, and Simon propose that problem-solving behavior might be explained, in part, by appeal to information processing. On this model, behavior is the thing to be explained, whereas information processing is among the factors that do the explaining. They repeat this idea more emphat- 
ically a bit later in their paper: "At this level of theorizing, an explanation of an observed behavior of the organism is provided by a program of primitive information processes that generates this behavior" (ibid.) This is a conception of information processing/cognition they shared with Chomsky. Further, it is a conception Skinner recognized as the mainstream conception, arguing, however, that it is misguided. ${ }^{4}$ Notice that, by proposing that behavior is distinct from information processing and that information processing is realized in the brain, Newell, Shaw, and Simon implicitly adopt what is sometimes described as the framework of "mechanistic explanation." They propose to explain the behavior of a whole organism primarily by appeal to the behavior of one of its components. This picture might be illustrated with a well-known image from Craver 2007. (See Figure 1.) In this scheme, $S \psi$-ing would be something like a participant in an experiment solving a problem, whereas, say, $\mathrm{x}_{3} \phi_{3}$-ing would be the brain processing information.

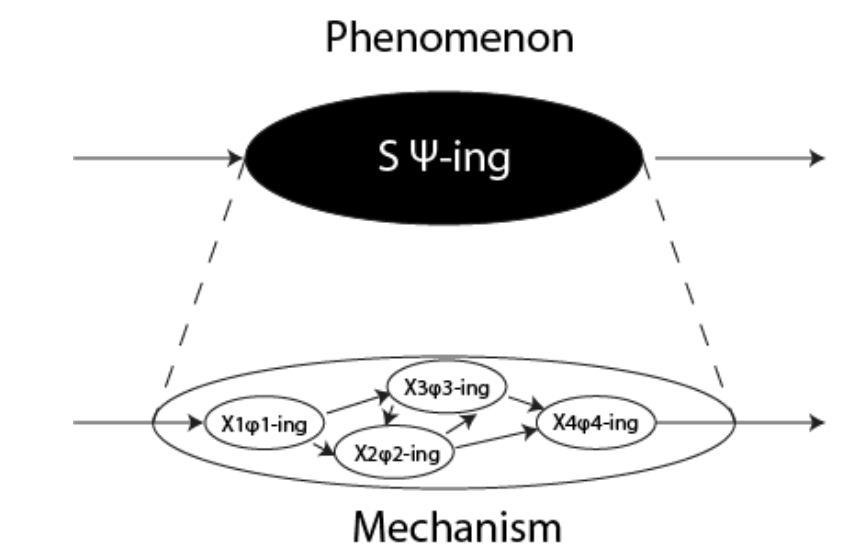

Figure 1. Schema for mechanistic explanation.

Redrawn from Craver 2007: 8, Figure 1.1.

Fourth, and finally, Newell, Shaw, and Simon embrace the traditional cognitive science focus on the role of information processing in the production of behavior, but they do not deny that there can be a role for sensory and motor activities in the production of behavior. We can describe this view by reference to the Craver schema. In the figure above, $\mathrm{x}_{1} \phi_{1}$-ing might be the eye performing a saccade, where $\mathrm{x}_{4} \phi_{4}$-ing might be writing with a pencil. Thus, they recognize that there are many component processes that conspire in the production of behavior, but indicate that they will limit their attention to a subset of these factors. The focus of their attention is methodological, not theoretical. In other words, even some of the earliest advocates of information processing psychology anticipated a day when psychologists might

\footnotetext{
${ }^{4}$ For a contemporary articulation of this picture, there is the Stanford Encyclopedia of Philosophy entry on cognitive science. (Thagard 2010).
} 
take up the issues concerning the contributions of sensory and motor factors to the production of behavior.

This traditional picture of the relationship between cognition and behaviorthat cognition is among the factors that might explain behavior-is rarely, if ever, explicitly discussed in the enactivist literature. Nevertheless, if one maintains that cognition is (a type of) behavior, one seems to be walking away from much of what cognitive science has been up to, namely, the study of putative endogenous contributions to the production of behavior. Nevertheless, enactivists $_{\mathrm{b}}$ have often seemed willing to do this, suggesting that a fresh start for cognitive science is in order. Setting aside questions about the wisdom of walking away from so much of the work that has been done in cognitive science, this paper will show some of the missteps this has engendered.

\section{Bourgine and Stewart 2004}

Recall Maturana's claim that "A cognitive system is a system whose organization defines a domain of interactions in which it can act with relevance to the maintenance of itself, and the process of cognition is the actual (inductive) acting or behaving in this domain." (Maturana and Varela 1980: 13). It is not entirely clear what is going on in this brief passage. Maturana apparently claims that the process of cognition is behaving in a domain-that cognition is a type of behavior-but one might have one's doubts that he genuinely proposes to identify cognition and behavior. How is this conception of cognition supposed to relate to the traditional conception, if at all? And, what are we to make of the character of this claim? Is it supposed to be a definition, a conceptual analysis, a theoretical hypothesis, or something else? It might well be read as a stipulative definition, but then again there are times when Maturana is prone to forceful pronouncements about empirical matters. Matters here are not that clear.

Some of the ambiguity in Maturana's text is eliminated from the account in Bourgine and Stewart 2004. The latter presentation is more deliberate and explicit about defining cognition as behavior and that this definition does not capture what is "ordinarily" meant by "cognition." Their proposal, therefore, at least looks more like a stipulative definition of "cognition." To provide their definition, they, first, define A interactions as those system-environment interactions that have consequences for the internal state of an organism and $B$ interactions as those system-environment interactions that have consequences for an organism's immediate environment or modify the relation of the organism to its environment. These terms then figure into a definition of "cognition": 
D-C1: A system is cognitive if and only if type A interactions serve to trigger type B interactions in a specific way, so as to satisfy a viability constraint.

Bourgine and Stewart explicitly decline to define what a "viability constraint" is, but the rough idea is that A interactions must trigger B interactions that are "good for" the system. This proposal seems to imply that systems are cognitive when stimuli provoke them to behave in ways that they are "good for" the system. And Bourgine and Stewart subsequently substantiate this interpretation:

It may be useful to illustrate this by examples of interactions such as falling down stairs, eating, or breathing (including the breathing of a poisonous but odorless gas). Ordinarily, such interactions are not considered as "cognitive." On the definition proposed here, they will not be cognitive unless the consequences for the internal state of the system are employed to trigger specific actions that promote the viability of the system. Thus, falling down stairs will be cognitive if but only if the fall triggers reactions such as a modification of muscle tone that limits the damage; and this does require specific sensory and motor organs. Similarly, eating is cognitive if but only if it triggers a reaction of satiety that prevents damage from overeating; breathing a poisonous gas is cognitive if but only if it triggers evasive action, which will require a specific sensory organ to detect the poison, and the resulting sensation to trigger an appropriate, coordinated motor response. (Bourgine and Stewart 2010: 338).

Bourgine and Stewart evidently concur with the cognitivist view that falling down stairs, eating, and breathing are not ordinarily considered to be cognitive (processes). Yet, they differ from cognitivists in their rationale for this assessment. For Bourgine and Stewart, it is only some instances of falling down stairs, eating, and breathing that are not to count as cognitive, namely, those in which there are no prophylactic effects, such as changes in muscle tone or satiety. For cognitivists, however, falling down, eating, and breathing are not, strictly speaking, cognitive processes at all; ${ }^{5}$ they are, at most, behavior. Moreover, they are likely to receive distinct behavioral analyses. By cognitivist lights, many instances of eating may count as cognitive behaviors insofar as they require cognitive processes in order to do things such as recognize food or to plan how to use knife and fork to obtain bite-sized pieces of food. By contrast, instances of breathing may not count as cognitive behaviors insofar as they do not involve cognitive processing. Humans typically breathe without thinking about it. Non-cognitive autonomic processes generally suffice for breathing. Finally, relatively few cases of falling down stairs will count as cognitive behaviors. Usually, gravity can do most of the work of tumbling someone down stairs without their really thinking about it.

\footnotetext{
${ }^{5}$ For clarification of the qualifier, "strictly speaking," recall the first point in the discussion of the passage from Newell, Shaw, and Simon 1958, in section 1 above.
} 
The point here, of course, is not to pin down precise frequencies or analyses of these cases, but merely to give a nod to the sorts of factors that will enter into cognitivist analyses and to contrast them with Bourgine and Stewart's analysis.

As we have just seen, the traditionalist might well claim that Bourgine and Stewart's theory is subject to counterexamples in which they call things that are not cognitive, cognitive. Falling down stairs is not a cognitive process, even though Bourgine and Stewart's theory says it is. A traditionalist can also easily imagine cases in which Bourgine and Stewart's theory would call cognitive processes "non-cognitive." So, imagine a person, Jane, looking out the window and seeing a cloudy day and thinking, "It looks like rain." This looks to be what Bourgine and Stewart would call an A interaction. Jane might then think, "Maybe I should take my umbrella. But, then again, I have a lot to carry today. Maybe I should just chance it and leave my umbrella at home," before she finally walks out the door. ${ }^{6}$ Jane's interior monologue and the walking out the door looks to be what Bourgine and Stewart would call a B interaction: it is a system-environment interaction that modifies the relation of Jane to her environment. But, now, suppose that a dramatic cloudburst drenches Jane and that this is not good for her. By Bourgine and Stewart's enactivism Jane's interior monologue was not a cognitive process, nor was there anything like a thought process underlying her interior monologue. This looks, to a cognitivist, like a counterexample to Bourgine and Stewart's theory in which a cognitive process is mistakenly labeled "non-cognitive.,"

At this point, one might propose that Bourgine and Stewart can simply stand by their stipulative definition of what a cognitive process is. Their theory does not capture traditional "intuitions," "theories," or-one might say, "false leads," but that is not what it is meant to do. Bourgine and Stewart, therefore, have a kind of theoretical "safety" in offering a stipulative definition of what

\footnotetext{
${ }^{6}$ Benny Shannon is an enactivist (though not an enactivist ${ }_{\mathrm{b}}$ ) for whom the study of such thought sequences is crucially important. (Cf., Shannon 2010.) As will emerge, Bourgine and Stewart's theory of cognition appears to have the consequence that some of the thought sequences that Shannon has studied will not be cognitive. Or, if one prefers, Bourgine and Stewart's theory of $\operatorname{cognition}_{b}$ appears to have the consequence that some of the thought sequences that Shannon has studied will not be cognitive ${ }_{b}$.

${ }^{7}$ This sort of scenario can be used to draw attention to another feature of Bourgine and Stewart's account. We cannot tell just from the occurrence of the A interaction and the B interaction whether or not a process is cognitive in Bourgine and Stewart's sense. Whether Jane's interior monologue was (indicative of) a cognitive process or not apparently depends on whether or not it actually rains and what impact this has on her. So, if it does not rain and this turns out to be good for Jane, say by sparing her the burden of carrying an unnecessary umbrella, then we have a cognitive process. Alternatively, if a downpour drenches Jane and this is not good for her, then the interior monologue would not be (indicative of) a cognitive process. Bourgine and Stewart's theory makes a process cognitive or non-cognitive based on contingent events that take place after the putative thought process.
} 
they mean by "cognition." The problem with this, however, is that when Stewart tries to use the theory to address traditional problems, he thereby misses his target.

Stewart claims that "There are two basic requirements for any paradigm in cognitive science: it must provide a genuine resolution of the mind-body problem, and it must provide for a genuine core articulation between a multiplicity of disciplines" (Stewart, 2010: 1). He then proposes a solution to the mindbody problem:

As discussed in Bourgine and Stewart 2004, we may define a system as "cognitive" if and only if it generates its actions, and the feedback sensations serve to guide actions, in a very specific way so as to maintain its autopoiesis and hence its very existence. With these definitions, "cognition" and "life" are fundamentally the same phenomena; and, in principle, the mind-matter problem is solved. (Stewart 2010: 1-3).

Thus, Stewart has proposed to solve the mind-body problem by a form of type identity theory: cognitive processes are biological processes (life processes). ${ }^{8}$ Then biological/life processes are, in turn, identified with physico-chemical processes (Cf., Stewart 2010: 203). So, it looks like Stewart and his enactivism strike squarely at one of the central issues in the philosophy of mind.

Appearances here are deceiving. If Stewart maintains that by "cognition" he does not mean what has traditionally been meant by cognition-if he does not correctly characterize what has traditionally been meant by "cognition" or "the mind," then he is not addressing the traditional mind-body problem. The traditional mind-body problem has not been concerned with how to relate falling down, eating, or breathing to biological or physico-chemical processes. Instead, the traditional mind-body problem concerns what is perhaps a cluster of problems, none of which centers on behaviors.

Notice that the mind-body problem as found in Descartes' philosophy is a question of how there could be causal interactions between an immaterial soul or mind and a material body. But, this is a question of how cognition, as traditionally construed, can interact with bodily processes. It is not a question of how cognition, construed as behavior, can interact with bodily processes. Stewart's "cognition" does not speak to the Cartesian version of the mindbody problem.

Kim 2005, however, observes that the Cartesian mind-body problem is not the contemporary mind-body problem found in the philosophy of mind and cog-

\footnotetext{
${ }^{8}$ One might well make the case that Stewart does not have a type identity theory solution but a functionalist solution. The difference probably does not make a difference here, since the weakness in Stewart's purported solution lies in his view that cognition is viable behavior. Maybe there are other enactivist ${ }_{b}$ tools for dealing with this portion of the traditional mind-body problem, but the Bourgine-Stewart theory of cognition alone will not suffice.
} 
nitive science. Instead, the contemporary mind-body problem appears to be a cluster of problems. One of these is a problem of mental causation. This problem, however, is not (at least in the first instance) about how bodily movements can be causally efficacious; it is about how internal states and processes, such as beliefs, desires, intentions, and decisions, could be among the causes of behavior. (Cf, Kim 2005: 7f). If Stewart is not thinking of cognitive states as internal causes of behavior, then he is not grappling with the mental causation portion of the mind-body problem. A second problem in the cluster of mind-body problems is the problem of consciousness. By consciousness, Kim has in mind qualitative features such as "the smell of the sea in a cool morning breeze, the lambent play of sunlight on brilliant autumn foliage, the fragrance of a field of lavender in bloom, and the vibrant, layered soundscape projected by a string quartet” (Kim 2005: 11). David Chalmers seems to have a similar thing in mind, though described with different examples: "the quality of deep blue, the sensation of middle C" (Chalmers 1995: 201). Insofar as Stewart's theory of "cognition” (or mind) does not capture what is traditionally meant, but constitutes a stipulative definition not tied to these cases, Stewart evidently fails to address the traditional mind-body problem. We might concede, if only for the sake of being agreeable, that Bourgine and Stewart do solve a version of the mind-body problem, namely, the version that shows how cognition defined in $\mathrm{D}-\mathrm{C} 1$ can be related to the body. But, even with that concession, it remains true that Stewart has not resolved the traditional mind-body problem(s). Instead, enactivism $b$ threatens to leave aside old issues for new issues.

So, to summarize this section, one might begin with the possibility that Bourgine and Stewart have proposed a definition of "cognition" that is meant to capture what has traditionally been thought of as cognition. Two sorts of counterexamples, however, challenge this view. 1) Falling down stairs is not a cognitive process; it is (at best) a cognitive behavior. 2) Interior monologues constitute, or are indicative of, cognitive processes even in cases where these interior monologues (or the processes underlying them) lead to bad outcomes for their possessors. In light of these considerations, it is perhaps better to interpret Bourgine and Stewart as not merely overthrowing the theoretical apparatus of cognitivism with its computational rules and representations. Instead, they also propose to overthrow the putative exemplars of cognition. Cognition is not one among many possible endogenous causal contributors to behavior; it is, instead, viable behavior. If, however, this is what Stewart is up to, then he appears to be walking away from many of the problems that cognitivism faced. For example, Stewart's identification of cognition and life does not solve the traditional mind-body problem. It solves, at best, a novel version of the mind-body problem. 


\section{Froese, Gershenson, and Rosenblueth 2013}

The hypothesis of extended cognition, in at least some of its earliest articulations, maintains that, while brains realize some cognitive processes, sometimes, under certain conditions, larger configurations of brain, body, and world also realize cognitive processes. This is the conception that was implicit in parts of Andy Clark and David Chalmers' seminal paper. (See Clark and Chalmers 1998.) It was the conception in play in the two "cognitive equivalence" arguments based on the play of the video game Tetris and the Inga-Otto thought experiment. Recall the description of three modes of Tetris play:

(1) A person sits in front of a computer screen which displays images of various two-dimensional geometric shapes and is asked to answer questions concerning the potential fit of such shapes into depicted "sockets". To assess fit, the person must mentally rotate the shapes to align them with the sockets.

(2) A person sits in front of a similar computer screen, but this time can choose either to physically rotate the image on the screen, by pressing a rotate button, or to mentally rotate the image as before. We can also suppose, not unrealistically, that some speed advantage accrues to the physical rotation operation.

(3) Sometime in the cyberpunk future, a person sits in front of a similar computer screen. This agent, however, has the benefit of a neural implant which can perform the rotation operation as fast as the computer in the previous example. The agent must still choose which internal resource to use (the implant or the good old fashioned mental rotation), as each resource makes different demands on attention and other concurrent brain activity. (Clark and Chalmers 1998: 7).

Case (1) looks to be a case of someone playing the video game using oldfashioned, brain-internal information processing. By contrast, (2) and (3) are supposed to be cognitively the same as (1) with the only difference between them being the material substrates that realize cognition. Next recall the Inga-Otto thought experiment. Inga has normal human memory, reads that there is an interesting exhibit at the Museum of Modern Art, and decides that she would like to see it. She thinks for a moment, recalls that it is on $53^{\text {rd }}$ St., then heads on her way. By contrast, Otto is suffering from the early stages of Alzheimer's Disease, so he has developed a system for maintaining information in a notebook. In this notebook, he has the address of the Museum of Modern Art. When he reads of the exhibit at the museum, he decides that he would like to see it. He then picks up his notebook, flips through it until he finds the address, then heads on his way. Clark and Chalmers claim that "in relevant respects the cases are entirely analogous: the notebook plays for Otto the same role that memory plays for Inga. The information in the notebook functions just like the information constituting an ordinary non-occurrent belief; it just happens that this information lies beyond the skin" (Clark and Chalmers 1998: 13). 
Whereas Clark and Chalmers sometimes offer "cognitive equivalence arguments” for extended cognition, Froese, Gershenson, and Rosenblueth 2013, propose a different path. They reject the idea that there is ever any brainbound, information processing type cognition of the sort that Inga was said to have. Instead, they adopt the enactivist ${ }_{b}$ view that cognition is (a type of) behavior: "cognition is primarily conceived of as a form of viable conduct by an agent in an environment" (Froese, Gershenson, and Rosenblueth 2013: 1). Thus, they take it that "[the Dynamical Systems Hypothesis] takes the notion of an extended mind as its starting point, rather than as a curious exception" (ibid.) Froese, Gershenson, and Rosenblueth, therefore, defend extended "cognition" in the sense of extended adaptive behavior. This path, however, does nothing to help the version of extended cognition in the Tetris and IngaOtto examples. Through those examples, Clark and Chalmers maintained the bold conclusion that what was once thought to have been realized only in the brain-a type of information processing - is, in fact, also sometimes realized in the brain, body, and world. Thus, Froese, Gershenson, and Rosenblueth, do not so much defend the hypothesis of extended cognition as leave it in favor of the hypothesis of extended viable conduct. ${ }^{9}$

Suppose, then, for the sake of argument that we follow Froese, Gershenson, and Rosenblueth and think about viable conduct. If so, then the further step to the view that such "cognition" is extended is trivial. "Conduct" is essentially another word for behavior and behavior typically is realized by brain, body, and world. In cases in which hammering in a nail is a viable behavior, it is apparently realized by cognitive, attentional, and motivational processes in the brain, along with the propagation of light in the eye, along with muscular processes in the arms, and contact between the hammer and a nail. Who would doubt that? Why would anyone doubt that? So, where the hypothesis of extended cognition is a controversial hypothesis, the hypothesis of extended viable conduct appears to be widely if not universally accepted. What makes the latter appear interesting is the enactivist $t_{b}$ terminology of using "cognition" as a term for viable conduct, where most cognitive scientists think of "cognition" as a term for cognition.

Froese, Gershenson, and Rosenblueth appear to appreciate that they cannot rely on the view that cognition is a form of viable conduct in order to argue for the extended cognition hypothesis. Therefore, they argue that "even if we accept [the] internalist starting point, a proper understanding of neuronal activity will force us to accept an extended view of the mind nonetheless." (Froese, Gershenson, and Rosenblueth 2013: 2). Given this concession, they propose two distinct arguments for extended cognition. There is an extensive

\footnotetext{
${ }^{9}$ If one prefers, one might say that Clark and Chalmers defend something more like extended $\operatorname{cognition}_{c}$, where Froese, Gershenson, and Rosenblueth defend extended cognition ${ }_{b}$. Again, the body of the text uses more standard terminology.
} 
discussion of an evolutionary robotics simulation, but the details of that are irrelevant to their arguments, so will not be reviewed.

Consider their two arguments in reverse order of their appearance. Their second argument is relatively simple:

6. The non-isolated [Continuous-Time Recurrent Neural Network]'s output is determined by its input, albeit mediated by its internal activity, while this input is determined by its motor output, albeit mediated by bodily and environmental (including social) activity.

7. It logically follows from the above that the non-isolated CTRNN's additional neural complexity is partially constituted by its own sensorimotor and social coupling. (Froese, Gershenson, and Rosenblueth 2013: 6). ${ }^{10}$

This is a relatively simple version of the infamous "coupling-constitution fallacy". ${ }^{11}$ Froese, Gershenson, and Rosenblueth's contention notwithstanding, the second sentence above does not logically follow from the first. In the most simplistic of coupling arguments, one might observe that a cognitive process $\mathrm{X}$ is causally influenced by a prima facie non-cognitive process $\mathrm{Y}$, then infer that the appearances of these processes are misleading. Instead, the entire Y-X process is a cognitive process. The Froese, Gershenson, and Rosenblueth example, however, is a bit more complicated, since there are supposed to be reciprocal causal connections wherein $\mathrm{Y}$ causally influences $\mathrm{X}$ and $\mathrm{X}$ causally influences Y.

Reciprocal causal connections, however, do not suffice to close the gap between causation and constitution. Suppose we accept the internalist view that the non-isolated CTRNN has some "neural complexity." The premise in 6 notes that the CTRNN output is causally determined by its input and its input is causally determined by its output. This is a causal premise. The conclusion, however, is that the "additional neural complexity" is constituted by the processes outside of the CTRNN. But, why go beyond thinking that the additional neural complexity is merely causally influenced by processes outside the CTRNN? There seems to be no warrant for the conclusion that the additional neural complexity is constituted by external processes. ${ }^{12}$ Note that, in a nor-

\footnotetext{
${ }^{10}$ Technically, the argument seems to be for extended "neural complexity," whatever that is. To make this relevant to the hypothesis of extended cognition, there would need to be some link between "neural complexity" and cognition and it is unclear what Froese, Gershenson, and Rosenblueth propose this link to be. They think that cognition is viable conduct, not neural complexity. And, traditional cognitivism takes cognition to be something like rule-governed, symbol manipulation or information processing, not "neural complexity." For present purposes, however, these idiosyncrasies can be set aside.

${ }^{11}$ Cf., e.g., Block 2005, Adams \& Aizawa 2008, Rupert 2009.

${ }^{12}$ Froese, Gershenson, and Rosenblueth seem to think that it matters whether the environment qualitatively changes the CTRNN implementing an artificial nervous system (ANS):
} 
mally functional HVAC system, the thermostat's output is determined by its input, albeit mediated by its internal activity, while its input is determined by its output, albeit mediated by environmental activity, such as the burning of natural gas in the furnace. Nevertheless, the change of shape of the bimetallic strip in the thermostat is still limited to the bimetallic strip.

Froese, Gershenson, and Rosenblueth's second argument relies on a distinction between the properties of parts and the properties of wholes and the idea that these properties are often qualitatively distinct. This is an idea that is quite familiar from the literature on mechanistic explanation and is illustrated in Figure 1, in section 1, above. The idea is that entities do things in virtue of their parts doing qualitatively distinct things. The idea also appears in the enactivism literature in the introduction to Hutchins 2010:

Distributed cognition is a framework for exploring the cognitive implications of the commonsense observation that in systems characterized by multiple levels of interacting elements, different properties may emerge at different levels of organization. Thus, a colony of social insects has different properties than any individual insect in the colony. At the level of organisms, bodies have different properties than organs, which have different properties than cells. In the realm of cognition, a neural circuit has different properties than any of the neurons in the circuit. The same can be said of a brain area with respect to the neural circuits that compose it, or of an entire brain with respect to the areas that interact within the brain. This is also true of the body/brain system with respect to either brain or body, and the world/body/brain system with respect to any of its parts. A system composed of a person in interaction with a cognitive artifact has different cognitive properties than those of the person alone. (Hutchins 2010: 425.)

Froese, Gershenson, and Rosenblueth, propose to avoid the couplingconstitution fallacy by appeal to something like this picture:

This critique is known as the "coupling-constitution fallacy". In this paper we respond to this reductionist challenge by using an evolutionary robotics ap-

our aim is to build a model of an embodied agent, whose artificial nervous system (ANS) has mathematical properties that are in principle impossible for it to have in isolation. The motivation for this criterion is the need to go beyond a demonstration of how an agent's situatedness within a sensorimotor loop modulates the internal activity of the ANS, but can transform the ANS into a qualitatively different kind of system altogether.

if an ANS with less than 3D is nonlinearly coupled with other non-chaotic systems, and its internal neural activity spontaneously becomes chaotic, then an explanation of this property as resulting from an extended process of interaction cannot be accused of committing the coupling-constitution fallacy. (Froese, Gershenson, and Rosenblueth 2013: 2).

So, their idea is that when this ANS interacts with the environment, it undergoes a qualitative shift. It becomes chaotic. But, what does this have to do with cognition? It isn't that becoming chaotic is the same as becoming cognitive, is it? And, what does this have to do with what does, or does not, constitute a cognitive process? Why not stick with the idea that interaction with the environment causes the ANS to become chaotic? 
proach to create a minimal model of two acoustically coupled agents. We demonstrate how the interaction process as a whole has properties that cannot be reduced to the contributions of the isolated agents. We also show that the neural dynamics of the coupled agents has formal properties that are inherently impossible for those neural networks in isolation. (Froese, Gershenson, and Rosenblueth 2013: 1).

From the perspective of the DSH, which proposes a distributed view of cognition as the default mode of cognition, there is no coupling-constitution fallacy because properties of the sensorimotor interaction process cannot be reduced to that of the isolated components. (Froese, Gershenson, and Rosenblueth 2013: 6).

It is a good strategy for the EMH to highlight that ongoing interaction between a cognitive agent and environment results in a novel, mutually encompassing process with new properties of its own. (Ibid.)

With this picture, the work-around for the coupling-constitution fallacy is easy. There are brain properties, bodily properties, environmental properties, and brain-body-environment properties. In other words, there are properties the brain has, properties the body has, properties the environment has, and properties a brain-body-environment system has. One does not need to say that it is interaction between brain, body, and world that converts bodily properties and environmental properties into new properties. Instead, a causal interaction between brainy, bodily, and environmental processes gives rise to a new, qualitatively distinct, emergent property that is cognitive. Problem averted.

Notice that, for this argument, one does not really need a whole lot of evolutionary robotics simulation. It depends primarily on the picture of mechanistic explanation according to which higher level properties are realized by properties of lower level individuals. All of that is fine. There is, however, one tacit premise that is the downfall of this reply to the coupling-constitution fallacy. Recall that Froese, Gershenson, and Rosenblueth proposed to begin with the internalist assumption that cognitive processes are realized by the brain. Recall that they wanted to show that "even if we accept [the] internalist starting point, a proper understanding of neuronal activity will force us to accept an extended view of the mind nonetheless.” (Froese, Gershenson, and Rosenblueth 2013: 2). But, if they concede that assumption, namely, that the brain properties are cognitive, then given their other premise that the properties of the brain-body-world system are qualitatively distinct from the properties of the brain, the body, and the world, then this gives us the beginnings of an argument that the properties of the brain-body-world system are not cognitive! The argument potentially backfires.

Of course, Froese, Gershenson, and Rosenblueth may be too quick to concede that brain properties are cognitive. Perhaps they should simply stick to their view that cognitive processes are instances of viable conduct. If they do that, 
however, then there really is no need for the coupling kinds of arguments at all. Clearly viable conduct is extended. As noted above, no one doubts that when hammering in a nail is a viable behavior, it is probably realized by cognitive, attentional, and motivational processes in the brain, along with the propagation of light in the eye, along with muscular processes in the arms, and physical processes in the hammer, nail, and wood. Once you have the view that cognition is a type of behavior, it is relatively smooth sailing to the conclusion that such "cognition" is extended.

So, the upshot of our discussion of Froese, Gershenson, and Rosenblueth's paper is three-fold. If they wish to defend the view that viable conduct is extended, then they are doing nothing to support one of the original versions of the idea that cognition is extended. They are not at all supporting the idea that some sort of information processing style of cognition is extended. Second, if they wish to defend the view that viable conduct is extended, then they are not defending a view that it seems anyone has ever doubted. The standard view in cognitive science is that conduct or behavior is extended. Third, the appeal to the framework of mechanistic explanation does nothing to avoid the problems of the coupling-constitution fallacy, unless one begins with the assumption that cognition is a property of a brain-body-world system. But, if one has that assumption, there is no need for further argumentation using the framework of mechanistic explanation. Properties of a brain-body-world system are clearly extended. What this suggests is that, by taking "cognition" to be a term for viable conduct, rather than for some endogenous causal contributor to the production of conduct, Froese, Gershenson, and Rosenblueth have marginalized their view from the concerns of extended cognition and the coupling-constitution fallacy.

\section{Hutto and Myin 2013}

From the earliest pages of their Radicalizing Enactivism, Daniel Hutto and Erik Myin challenge the view that all cognition requires representations. Their alternative is Radically Enactive (or Embodied) Cognition (REC):

The most radical versions of these approaches are marked by their uncompromising and thoroughgoing rejection of intellectualism about the basic nature of mind, abandoning the idea that all mentality involves or implies content. Call this-the view we defend-Radically Enactive (or Embodied) Cognition-REC for short. (Hutto and Myin 2013: 1)

Embodied ways of thinking reject the familiar explanatory framework of orthodox cognitive science in favor of alternative platforms. Adherents of such views deny that the best way to explain cognition is to posit the construction of internal representational models (ibid.: 2) 
Defenders of REC argue that the usual suspects-representation and computation-are not definitive of, and do not form the basis of, all mentality. (ibid.: 3).

Notice in these claims they write of rejecting "the familiar explanatory framework of cognitive science" and "the usual suspects-representation and computation." The familiar view, however, is that all cognition, understood as one of the internal, brain-realized causes of behavior, involves representation. The tradition does not, however, maintain that (adaptive) behavior involves representation. Plants, for example, might display adaptive behaviors, such as phototropism, without deploying representations to do this. Such cases would be cases in which plants produce behaviors that are not cognitive behaviors. They are not behaviors that are produced, in part, through cognitive processes.

By contrast, when Hutto and Myin stake out their own view about cognition, they apparently have a different conception of cognition. They use "basic cognition" as a phrase to describe what sounds like behavior: "We restrict our ambitions to promoting REC, calling upon strong versions of two theses. We dub these the Embodiment Thesis and the Developmental-Explanatory Thesis. The former equates basic cognition with concrete spatio-temporally extended patterns of dynamic interaction between organisms and their environments." (Hutton and Myin 2013: 5). For Hutto and Myin, "basic cognition" is concrete spatio-temporally extended patterns of dynamic interaction between organisms and their environments. This is a description of what traditional cognitive science would call "behavior." They also seem to use "cognition" as a term for behavior. In the early pages of their book, they write that "proponents of Enactive and Embodied ways of thinking reject the familiar explanatory framework of orthodox cognitive science in favor of alternative platforms. Adherents of such views deny that the best way to explain cognition is to posit the construction of internal representational models built on the basis of retrieved informational content." (Hutto and Myin: 2). It is somewhat odd to say that traditional approaches explain cognition by positing the construction of internal representational models. As we saw with Newell, Shaw, and Simon, the traditional approach is to explain behavior by positing representations, among other things. This oddity disappears, however, if we understand them to use "cognition" as a word for behavior. Second, they claim that "Enactivism is inspired by the insight that the embedded and embodied activity of living beings provides the right model for understanding minds." (ibid.: 4). Embedded and embodied activity, which sounds like another description of behavior, probably would be a very good model for the mind, if the mental were the behavioral.

The stage is now set for traditional cognitive science to go about its business of studying cognition that purportedly must involve representations, where enactivism $_{b}$ goes about its business of studying behavior which need not involve 
representations. Nevertheless, things do not go so smoothly. Hutto and Myin's use of the phrase "basic cognition" muddies what might otherwise be a very simple argument. Hutto and Myin want to challenge the standard view that all cognition requires representation and one way they wish to do this is by providing counterexamples. As a point of logic, they evidently want a case, or two, in which there is cognition without representation. But, instead of cases in which there is cognition without representation, they only provide cases of their "basic cognition" without representation. That is, they only provide cases of behavior without representation.

Hutto and Myin's two putative counterexamples are Rodney Brooks' behaviorbased robots and Barbara Webb's models of cricket phonotaxis. (See Brooks 1997, Webb 1994, 1996.) Even as Hutto and Myin describe the example, Brooks' robots show only cases of behavior that does not require representation: 'Brooks' first-generation behavior-based robots, and those that followed, succeed precisely because their behaviors are directly guided by continuous, temporally extended interactions with aspects of their environments, rather than being based on represented internal knowledge about those domains (knowledge that would presumably be stored somewhere in the robots' innards)." (ibid.: 42). Such an analysis is irrelevant to mainstream cognitive science, since it only shows that behavior does not require representation, not that cognition does not require representation. Thinking of "cognition" and "behavior" as referring to the same thing seems to mislead Hutto and Myin. The same oversight appears in their analysis of Webb's models of crickets. By their own analysis, "Webb's (1994, 1996) work on cricket phonotaxis offers a vivid example of a model of how successful navigation takes place in the wild, apparently without the need for representations or their manipulation. ... In other words, the capacity of these animals to adjust their behavior when successfully locating mates requires them to engage in a continuous interactive process of engagement with the environment.” (ibid.: 43). So, by Hutto and Myin's own analysis, these are only cases of behavior without representation; not cases of cognition without representation. ${ }^{13}$

What the foregoing suggests is that enactivist ${ }_{b}$ terminology confuses even Hutto and Myin. It complicates what should be a simple exercise is trying to generate a counterexample to the claim that all cognition requires representation. Rather than offering examples of cognition that do not involve representation, they only offer examples of behavior that do not involve representation. This, of course, only shows that one argument for REC has not worked. It does not show that the argument cannot be fixed, as by revisiting the examples and

\footnotetext{
${ }^{13}$ AUTHOR shows how Chemero 2009, makes much the same sort of mistake in thinking that models in Beer 2003, and van Rooij, Bongers, and Haselager 2002, "show how radical embodied cognitive science can explain cognition as the unfolding of a brain-body-environment system, and not as mental gymnastics” (Chemero 2009: 43).
} 
showing how they have internal mechanisms that are plausibly cognitive, but which nevertheless contain no representations. Nor does it show that there are no other arguments for REC that might work. ${ }^{14}$ Much less does it show that REC is false. As billed at the outset, the goal here is to clarify some of missteps in the enactivist $t_{b}$ revolution. The point of clarification here is that Hutto and Myin's formulation of an argument based on the Brooks and Webb models do not work as billed.

\section{Not all Enactivists are Enactivists}

The goal of this paper has not been to critique the whole of the enactivist program. As is often noted, enactivism is still in its formative stages and many ideas remain to be worked out on many different topics. Some authors associated with enactivism do not seem to be concerned with cognition at all. So, for example, Barbaras 2010, which was included in Stewart, Gapenne, and Di Paolo's Enaction anthology, wrote about life and metabolism and barely mentioned cognition. Moreover, as is to be emphasized now, not all enactivists are enactivists $_{\mathrm{b}}$. One can say this, but its force might be better appreciated if we describe enactivists who are not enactivists $\mathrm{b}_{\mathrm{b}}$.

Perhaps one of the more significant examples is that, some years ago, Stewart seems not to have been an enactivist ${ }_{b}{ }^{15}$ In the abstract to a 1996 paper, Stewart writes, "In contemporary cognitive science, there are two distinct paradigms with contrasting definitions of cognition. The computational theory of mind is based on the syntaxical manipulation of symbolic representations; this paradigm is objectivist because the postulate of a unique independent reality is necessary as a referential basis for semantic grounding of the symbols. The alternative 'constructivist' paradigm is based on the biological metaphor 'cognition = life' and programmatically follows the evolution of cognition from bacteria to civilized humans; it is non-objectivist." (Stewart 1996: 311). Rather than articulating two distinct accounts of what cognition is, however, Stewart appears to be thinking of something along the lines of what philosophers might interpret as a distinction between a form of realism and a form of anti-realism: "In an objectivist [computationalist] perspective, cognition is the subjective representation of an ontologically independent objective reality. In a constructivist perspective, based on the biological metaphor 'cognition = life', the clear point of contrast is that the subject and the object of knowledge

\footnotetext{
${ }^{14}$ In fact, Hutto and Myin spend a lot of time arguing that causal and informational approaches to naturalizing content have failed, hence that we therefore have some reason to think we should abandon the hypothesis that all cognition requires representation. This argument is untouched by the foregoing.

${ }^{15}$ Shannon 2010, also takes an enactivist, but not an enactivist ${ }_{b}$ position. Limitations of space preclude a discussion of this.
} 
are not independent but are mutually constitutive” (Stewart 1996: 316.) ${ }^{16}$ Stewart's "objectivism" and "constructivism" seem to be versions of realism and anti-realism.

In fact, it appears that at two points in this earlier paper Stewart understands cognition in a more traditional way, not as behavior, but as an internal mechanism that contributes to the production of behavior. As the first point, there is his commentary regarding what appear to be (cognitive) mechanisms by which animals can cancel out the effects of bodily movements in order to perceive organism independent objects.

Animals with a central nervous system have the capacity to distinguish within their own cognitive repertoire between modifications of their sensory input which are the immediate consequence of their own actions, and modifications which are not so caused. For example, when an animal moves its eyes, the retinal image (and hence the stimulation of the retinal cones) is modified, but a mammal does not usually confuse this movement with the movement of an object in its environment. The construction of perceptual invariants on the basis of motor-sensory correlations of this sort is thus at the basis of the emergence of a 'stable external world' populated by 'objects' which exist as such in the cognitive repertoire of the organism itself. ... Bacteria (or trees), for example, are quite incapable of cognitive feats of this sort. (Stewart 1996: 320).

Here it is at least possible that Stewart conceives of there being cognitive mechanisms that enable (some) animals, but not bacteria and trees, to avoid confusing the effects of self-movement with the effects of object movement. In fact, these different mechanisms might be just the kinds of differences between (some) animals, on the one hand, and trees and bacteria, on the other, that cognitivists would contend mark the difference between cognitive and non-cognitive processes. As a second point, Stewart notes that

\footnotetext{
${ }^{16}$ It is indicative of the cross-currents in enactivism that Hutchins 2010, was part of the Stewart, Gapenne, and Di Paolo Enaction anthology, but also apparently endorses the kind of computational theory of mind that Stewart would reject as "objectivist". Hutchins describes computational transformations on representations of what appear to be navigator-independent features of the world:
}

Two successive positions of a ship are plotted on a three-minute interval. Suppose the distance between them is 1500 yards. The navigator computes ship's speed to be 15 knots by doing the following: "The distance between the fix positions on the chart is spanned with the dividers and transferred to the yard scale. There, with one tip of the divider on 0 , the other falls on the scale at a tick mark labeled 1500 . The representation in which the answer is obvious is simply one in which the navigator looks at the yard-scale label and ignores the two trailing zeros" (Hutchins 1995..., 151-152). In this analysis, high-level cognitive functions were seen to be realized in the transformation and propagation of representational states. The span between the fix positions on the chart is a representational state that is transformed into a span on the dividers. This representational state is then transformed into a span on the yard scale. Finally, the span on the yard scale is transformed into the answer by reading the label on the designated tick mark in a particular way. (Hutchins 2010: 427).

Hutchins, thus, also appears to be an enactivist, but not an enactivist ${ }_{b}$. 
Armed with representations of this sort, an organism can set itself a 'goal' (expressed in terms of a desired perceptual configuration), and then by purely mental activity (without having to take the risks involved in proceeding by trial and error by actually acting in the world) elaborate a sequence of actions which, according to these representations, can be expected to achieve that goal (Stewart 2010: 320).

Here it looks likes there is a "purely mental activity" independent of actually acting in the world, i.e., independent of physical behavior in the world. So, the discussion in Stewart, 1996, suggests that one can be an enactivist without being an enactivist ${ }_{\mathrm{b}}$.

\section{Conclusion}

At the heart of this paper is the observation that some enactivists do not mean by "cognition" what traditionalists have meant by "cognition." There are, if you will, two concepts of cognition in play, a traditional concept and an enactivist concept. This observation would seem to be entirely unproblematic. Moreover, it would seem to be entirely unproblematic to note that some enactivists use "cognition" to describe (a kind of) behavior. These enactivists maintain that cognition is (viable) behavior. These enactivists are enactivists $_{\mathrm{b}}$. This choice of terminology-or this way of theorizing, if you willhowever, looks to be misleading. Moreover, it is not misleading just for traditionalists. It is misleading for even some enactivist $\mathrm{s}_{\mathrm{b}}$. By adopting a new conception of cognition-by thinking of behavior and cognition as the same thing-enactivist $\mathrm{s}_{\mathrm{b}}$ sometimes overlook ways in which they have detached themselves from the traditions of cognitive science. Enactivists are generally happy to break with these traditions, but there are also times when this break is not as complete as it should be. There are times when they try to engage with mainstream cognitive science, but are hampered by the steps they have already taken to break with tradition. One cannot solve the traditional mindbody problem, if one is not dealing with (something near enough to) the traditional conception of the mind. One might dissolve the problem or abandon the problem, if one rejects the traditional concept, but one cannot solve it. One cannot argue that cognition is embodied and extended, by observing that behavior is embodied or extended. And, one cannot show that not all cognition involves representation by providing instances of behavior that do not involve representation. None of these observations undermines the enactivist $_{\mathrm{b}}$ approach, much less any other enactivist approaches. They merely draw attention to some missteps in the evolution of enactivism ${ }_{b}$. Perhaps the safest route for enactivists $\mathrm{b}_{\mathrm{b}}$ is simply to make a clean break with traditional views. Perhaps enactivists ${ }_{b}$ should walk away from traditional views and leave them to their own devices. 


\section{References}

Adams, F., and Aizawa, K. 2008. The Bounds of Cognition. Malden. MA: Wiley-Blackwell.

Aizawa, K. 2014. What is this cognition that is supposed to be embodied? Philosophical Psychology. DOI:10.1080/09515089.2013.875280.

Barbaras, R. 2010. Life and exteriority: The problem of metabolism. E. Di Paolo, O. Gapenne and J. Stewart, eds. Enaction: Toward a new paradigm for cognitive science: 89-122). Cambridge, MA: MIT Press.

Block, N. 2005. Review of Alva Noë, Action in Perception. Journal of Philosophy, 102(5): 259-272.

Bourgine, P., and Stewart, J. 2004. Autopoiesis and cognition. Artificial life, 10(3), 327345.

Brooks, R. A. 1997. Intelligence without representation. J. Haugeland, ed. Mind design II: 395-420. Cambridge, MA: MIT Press.

Calvo, P., and Keijzer, F. 2008. Cognition in plants. F. Baluska, ed. Plant-Environment Interactions: From Sensory Plant Biology to Active Plant Behavior : 247-266. Berlin: Springer Verlag.

Chalmers, D. 1995. Facing up to the problem of consciousness. Journal of Consciousness Studies, 2(3): 200-219.

Chemero, A. 2009. Radical Embodied Cognitive Science. Cambridge, MA: MIT Press.

Chomsky, N. 1959. A review of Verbal Behavior. Language, 35(1): 26-58.

Clark, A. 2010. Coupling, constitution and the cognitive kind: A reply to Adams and Aizawa. R. Menary, ed. The extended mind: 81-99. Cambridge, MA: MIT Press.

Clark, A., and Chalmers, D. 1998. The extended mind. Analysis, 58(1): 7-19.

Craver, C. 2007. Explaining the Brain. Oxford: Oxford University Press.

Froese, T., Gershenson, C., and Rosenblueth, D. 2013. The Dynamically Extended Mind-A Minimal Modeling Case Study. arXiv preprint arXiv:1305.1958.

Di Paolo, E., Rohde, M., and De Jaegher, H. 2010. Horizons for the enactive mind: Values, social interaction, and play. E. Di Paolo, O. Gapenne and J. Stewart, eds. Enaction: Towards a New Paradigm for Cognitive Science: 33-87.

Haugeland, J. 1998. Mind embodied and embedded. Having Thought: 207-237. Cambridge, MA: Harvard University Press.

Hutchins, E. 2010. Enaction, imagination, and insight. J. Stewart, O. Gapenne and E. Di Paolo, eds. Enaction: Towards a New Paradigm for Cognitive Science: 425-450. Cambridge, MA: MIT Press.

Hutchins, E. 1995. Cognition in the Wild. Cambridge, MA: MIT Press.

Hutto, D. D., and Myin, E. 2012. Radicalising Enactivism. Cambridge, MA: The MIT Press. 
Kim, J. 2005. Physicalism, or Something Near Enough. Princeton, NJ: Princeton University Press.

Maturana, H. R., and Varela, F. J. 1980. Autopoiesis and Cognition: The Realization of the Living. Dordrecht: Springer.

Newell, A., Shaw, J. C., and Simon, H. A. 1958. Elements of a theory of human problem solving. Psychological Review, 65(3): 151-66.

Noë, A. 2004. Action in Perception. Cambridge, MA: MIT Press.

Rupert, R. 2009. Cognitive Systems and the Extended Mind. New York: Oxford University Press.

Shannon, B. 2010. Toward a phenomenological psychology of the conscious. J. Stewart, O. Gapenne and E. Di Paolo, eds. Enaction: Toward a New Paradigm for Cognitive Science: 387-424. Cambridge, MA: MIT Press.

Skinner, B.F. 1957. Verbal Behavior. New York, NY: Appleton-Century-Crofts.

Stewart, J. 1996. Cognition = life: Implications for higher-level cognition. Behavioural Processes, 35(1): 311-326.

Stewart, J. 2010. Foundational issues in enaction as a paradigm for cognitive science: From the origin of life to consciousness and writing. J. Stewart, O. Gapenne and E. Di Paolo, eds. Enaction: Towards a New Paradigm for Cognitive Science: 1-31. Cambridge, MA: MIT Press.

Stewart, J, Gapenne, O, \& Di Paolo, EA. 2010. Introduction. J. Stewart, O. Gapenne \& E. Di Paolo, eds. Enaction: Toward a New Paradigm for Cognitive Science: viixvii.

Thagard: 2010. Cognitive science. Retrieved November 24, 2013, 2013, from http://www.science.uva.nl/ seop/entries/cognitive-science/

Varela, F., Thompson, E., and Rosch, E. 1991. The Embodied Mind. Cambridge, MA: MIT Press.

Webb, B. 1994. Robotic experiments in cricket phonotaxis. D. Cliff: Husbands, M. J. A. and S. W. Wilson, eds. From Animals to Animats 3: Proceedings of the Third International Conference on Simulation of Adaptive Behavior: 45-54. Cambridge, MA: MIT Press.

Webb, B. 1996. A cricket robot. Scientific American, 275(6): 94-99. 